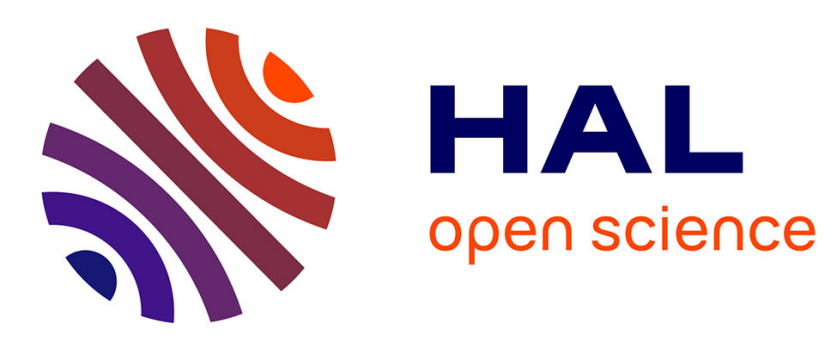

\title{
Foam rheology at large deformation
}

\author{
J.-C Géminard, J. C Pastenes, F. Melo
}

\section{To cite this version:}

J.-C Géminard, J. C Pastenes, F. Melo. Foam rheology at large deformation. Physical Review E , 2018, 97 (4), pp.42601 - 42601. 10.1103/PhysRevE.97.042601 . hal-01791427

\section{HAL Id: hal-01791427 \\ https://hal.science/hal-01791427}

Submitted on 14 May 2018

HAL is a multi-disciplinary open access archive for the deposit and dissemination of scientific research documents, whether they are published or not. The documents may come from teaching and research institutions in France or abroad, or from public or private research centers.
L'archive ouverte pluridisciplinaire HAL, est destinée au dépôt et à la diffusion de documents scientifiques de niveau recherche, publiés ou non, émanant des établissements d'enseignement et de recherche français ou étrangers, des laboratoires publics ou privés. 


\title{
Foam rheology at large deformation
}

\author{
J.-C. Géminard* \\ Université Lyon, Ens de Lyon, Université Claude Bernard, CNRS, Laboratoire de Physique, Lyon F-69342, France \\ J. C. Pastenes and F. Melo ${ }^{\dagger}$ \\ Departamento de Física Universidad de Santiago de Chile and SMAT-C, Avenida Ecuador 3493, Estación Central 9170124, Santiago, Chile
}

(Received 16 November 2017; published 4 April 2018)

\begin{abstract}
Large deformations are prone to cause irreversible changes in materials structure, generally leading to either material hardening or softening. Aqueous foam is a metastable disordered structure of densely packed gas bubbles. We report on the mechanical response of a foam layer subjected to quasistatic periodic shear at large amplitude. We observe that, upon increasing shear, the shear stress follows a universal curve that is nearly exponential and tends to an asymptotic stress value interpreted as the critical yield stress at which the foam structure is completely remodeled. Relevant trends of the foam mechanical response to cycling are mathematically reproduced through a simple law accounting for the amount of plastic deformation upon increasing stress. This view provides a natural interpretation to stress hardening in foams, demonstrating that plastic effects are present in this material even for minute deformation.
\end{abstract}

DOI: 10.1103/PhysRevE.97.042601

\section{INTRODUCTION}

While the elastic mechanical response of materials underlies on the relevant structures capable of sustaining reversible deformations, the plastic response is strongly determined by the concentration of defectlike structures, and of their distribution, proliferation, and mobility. In the nonlinear regime, besides the motion of defects, high deformations may produce irreversible structural changes, such as the proliferation of shear transformation zones (STZ) in amorphous materials [1], the progressive elimination of weaker contacts between grains in granular matter [2], or the reorganization of bundles in the actin network [3]. Prior material rupture and characteristic material softening or hardening upon cyclic deformation are frequently found independently of the material nature [4]. The best known example of material softening upon periodic stress was observed for filled rubbers by Mullins early in 1940 [5,6]. Since then, different soft and hard materials displayed significant softening and a striking capacity to print the history of the applied stress within their internal structure [4]. Moreover, it was recently suggested that Mullins-like softening, in materials ranging from single biopolymers over cells to model tissues, is a consequence of the presence of diverse mechanisms of energy dissipation, such as protein unfolding or sacrificial bonds [7]. While less frequent in soft materials, hardening has been recently observed in long-chain branched polymer solutions under shear [8], in granular matter [9], in foams [10], and in reconstituted networks of crosslinked, bundled, actin filaments [3] under periodic shear.

Despite fundamental differences at the microstructure level, evidence indicates that hardening and weakening are clear

\footnotetext{
*jean-christophe.geminard@ens-lyon.fr

${ }^{\dagger}$ francisco.melo@usach.cl
}

manifestations of microstructure remodeling driven by the applied stress. However, the common features of the remodeling processes occurring in structural networks of different nature and scale are still elusive, although valuable insight is available for polymers and biopolymers $[7,11,12]$. Foam has served as a model system for the study of plasticity of soft amorphous materials [13] and of its consequences such as stress relaxation [14], shear banding [15-17], and jamming [18]. However, most rheological studies of foam are currently performed at small deformation, while attention is rather focused on the viscoelastic properties at increasing shear rates [19].

Here we present experiments on foam layers subjected to quasistatic shear cycles, aiming at elucidating the response in the highly nonlinear regime. We observe that, upon increasing strain, the stress follows a universal curve and tends almost exponentially to an asymptotic value, that can be interpreted as the critical stress at which the foam structure is completely remodeled - the yield stress. As the strain direction is reversed, a similar behavior is observed; the shear stress tends to the same yield stress value but with opposite sign. All trends of cycling can be mathematically reproduced by a simple law that relates the ratio of the plastic deformation to the total deformation and the ratio of the external stress to the yield stress. This law can be deduced by assuming simple conservation rules for the distribution of active defects responsible for foam plasticity, and the complete refreshing of the foam structure for shear inversion. Thus, high amplitude rheological tests provide a useful tool to simply assess the main rheological properties of foams at very large strain, thus in a regime relevant to applications [20,21].

\section{EXPERIMENTAL SETUP AND PROTOCOL}

The experimental setup is a plane rheometer consisting of two parallel plates subjecting the sample in the gap between 


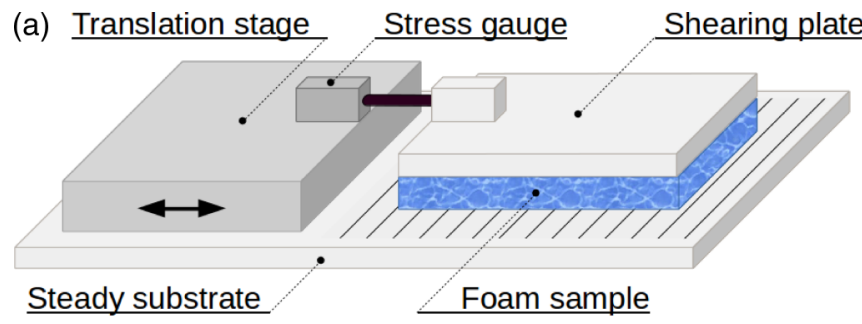
Steady substrate

(b)

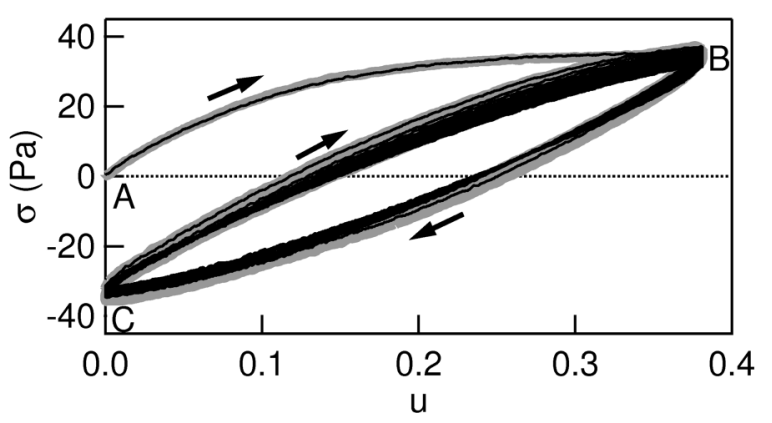

FIG. 1. (a) Sketch of the experimental setup: A thin layer of foam is deposited onto the horizontal bottom plate of the rheometer. The upper plate of the rheometer is periodically displaced along the $x$ axis by means of a motorized translation stage. Strain gauge provides the shear force. Layer thickness is fixed to $8 \mathrm{~mm}$. (b) Typical shear stress $\sigma$ vs shear strain $u$ for cycles of maximum strain, $u_{m}=0.375$. Loading starts at point A. Upon cycling, the stress tends to a limiting cycle between points $\mathrm{B}$ and $\mathrm{C}$ where the shear direction is reversed. Arrows show the cycling direction (velocity of the upper plate: grey, $0.3 \mathrm{~mm} / \mathrm{s}$; black, $0.5 \mathrm{~mm} / \mathrm{s}$ ).

them to a periodic simple shear [Fig. 1(a)]. The lower plate is kept fixed whereas a controlled micropositioning system (MTS25-Z8, Thorlab, Newton NJ) imposes a periodic linear motion to the upper plate (PMMA, $40 \mathrm{~mm}$ wide, $60 \mathrm{~mm}$ long, and $5 \mathrm{~mm}$ thick). A force cell (Futek, LSB200 FSH02534, Irvine, CA, USA) is used to measure the shear force as function of deformation with a sensitivity of $10 \mu \mathrm{N}$. Parallel groves on both shearing surfaces, of about $0.5 \mathrm{~mm}$ depth and $1.5 \mathrm{~mm}$ apart, oriented perpendicularly to the shear direction, avoid sliding at the walls.

In our experiments, foam consists of Gillette shaving cream (Gillette ${ }^{\circledR}$, Foamy ${ }^{\circledR}$, Regular), selected for our study because it has been used in many rheological previous works reported in the literature [19] and its properties have been relatively well characterized. The samples are prepared to fit exactly in the gap preventing lateral excess. To do so, foam is poured in a rectangular mold machined in a 8 -mm-thick acrylic plate (PMMA) leaning on the bottom surface. Once the mold is removed, a rectangular sample of foam $\left(40 \times 60 \mathrm{~mm}^{2}\right)$, having a well-defined thickness, is obtained. Image analysis of the free surface reveals a typical bubble size of about $(60 \pm 20) \mu \mathrm{m}$. We know from the literature that the gas volume fraction is about $92 \%$. The shearing upper plate is then gently deposited on top using a three axis micropositioning system (DT12XYZ/M Thorlab, Newton, NJ). During upper plate positioning some compression occurs such that the sample thickness is decreased by a few tenths of mm. Note, however, that the material of the layer does not significantly flow during the preparation. The whole setup is maintained at constant relative humidity,
$R_{H}=(42 \pm 2) \%$, and temperature $T=(23 \pm 1){ }^{\circ} \mathrm{C}$, in order to ensure well-defined experimental conditions and to control the aging of the material. Prior to any test, the sample is maintained at constant external conditions for $5 \mathrm{~min}$. Reproducibility of our experimental results proves that, under these conditions, the phenomenon we report on is not significantly altered, neither by the variability of the protocol used to prepare the layer nor by the natural aging of the material.

\section{SHEAR STRESS MEASUREMENTS}

Starting from a freshly prepared sample, we impose periodic shear, one cycle consisting of moving the upper plate back and forth at constant velocity. We denote by $u_{m}$ the maximum of the imposed shear deformation. After having checked that the results were not significantly altered by changes of the velocity at least up to $0.7 \mathrm{~mm} / \mathrm{s}$ (Fig. 1), we report results obtained at $0.1 \mathrm{~mm} / \mathrm{s}$. Upon cycling, the typical behavior of the stress $\sigma$ as a function of the strain $u$ is as follows [Fig. 1(b)]. During the first half cycle (from A to B), the stress exhibits a first loading curve. When the direction of the deformation is reversed (in $\mathrm{B})$, the stress decreases following a curve of inverted curvature toward point $\mathrm{C}$ in which the imposed deformation is again zero. During the next half cycle (from $\mathrm{C}$ to $\mathrm{B}$ ), the stress again increases, following a curve whose curvature is again inverted. After a limited number of imposed cycles, $\sigma$ vs $u$ tends to a limit cycle. As revealed by the interpolation [thick gray line in Fig. 2(a)] of the first half cycle (from A to B), the stress $\sigma$ depends almost exponentially on the strain $u$. A similar stress-strain curve (for the first loading) accounting for foam plasticity is observed in two-dimensional (2D) numerical simulations in relatively large systems [22]. Early description of the mechanisms of stress relaxation in foams, developed by Weaire and Kermode [23,24], also concerned numerically foam under periodic deformations. However, to our knowledge the complete response to shear cycling has not been fully addressed.

In order to provide a framework for our data analysis, we develop a simple, heuristic model, based on the a simple constitutive law. We first assume that any, imposed, total deformation $u$ of the material can be decomposed in an elastic $\epsilon$ and a plastic $v$ deformation, such that $u=\epsilon+v$. Such an assumption is not obvious but, as we shall see, our experimental results are well accounted for using the simplest linear decomposition, which suggests that the role of the nonlinearities is not important at this level of description. By definition, we have $\sigma=\epsilon / G$ where $G$ is the elastic shear modulus. Second, we assume that the material responds with gradual plastic deformation to the applied stress and that there exists a well-defined maximum stress $\sigma_{\infty}$ that the material can sustain. Thus, for $\sigma=\sigma_{\infty}$, any further increase of the total deformation $d u$ induces a plastic deformation $d v=d u$. In contrast, we can guess that any plastic deformation requires that the system is minimally under stress, such that $d v=0$ in the limit $\sigma=0$. Assuming that this holds true regardless of the sign of the stress, we write $d v=f\left(\frac{\sigma}{\sigma_{\infty}}\right) d u$ where $f$ is an even function satisfying the conditions $f(0)=0$ and $f( \pm 1)=1$. However, inspection of experimental data indicates that a good candidate for this function is simply $f\left(\frac{\sigma}{\sigma_{\infty}}\right)=\left|\frac{\sigma}{\sigma_{\infty}}\right|$. Indeed, given that the total 

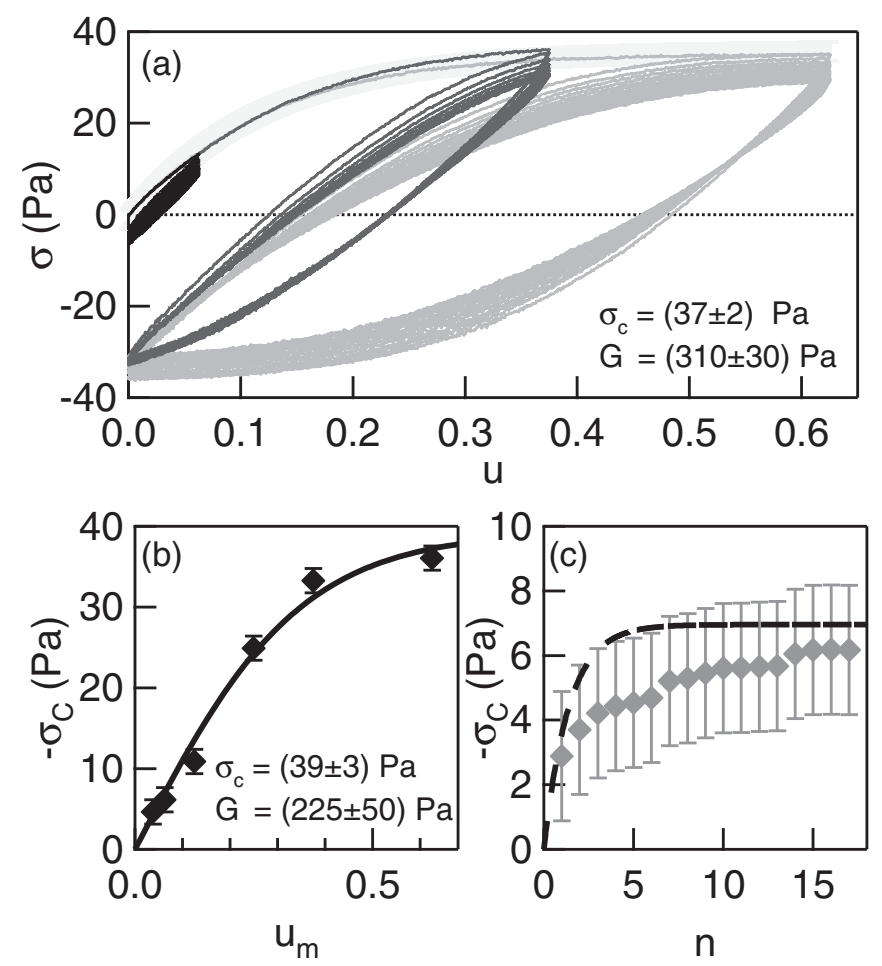

FIG. 2. (a) Shear cycles for increasing amplitude $u_{m}$. Starting at $\sigma=0$ and $u=0$, loading curves (A to B in Fig. 1) follow a universal nearly exponential law, underlined by the thick, light gray line. This line is the fit to the data using Eq. (1) with $\sigma_{\infty}=37 \mathrm{~Pa}$ and $G=$ $310 \mathrm{~Pa}$, the line thickness indicating fit errors (black: $u_{m}=0.0625$; dark gray: $u_{m}=0.375$; gray: $u_{m}=0.625$ ). (b) Evolution of shear stress at point $\mathrm{C}$ (Fig. 1) for the limit cycle as function of $u_{m}$. The solid line is the interpolation to Eq. (3) with $\sigma_{\infty}=39 \mathrm{~Pa}$ and $G=$ $225 \mathrm{~Pa}$. (c) Evolution of the stress at point $\mathrm{C}$ with number of cycles for $u_{m}=0.0625$. The dashed line represents the exponential dependence as predicted by Eq. (2) without adjustable parameter. The values $\sigma_{\infty}=$ $39 \mathrm{~Pa}$ and $G=225 \mathrm{~Pa}$ are taken from the previous fit.

deformation writes $d u=\frac{\sigma}{G}+d v$, such a function $f$ leads to

$$
\sigma= \pm \sigma_{\infty}\left[1-A_{ \pm} \exp \left(\mp \frac{G}{\sigma_{\infty}} u\right)\right]
$$

upon increasing $(d u>0,+$ sign) or decreasing $(d u<0$, sign) total deformation. The (positive) constants $A_{ \pm}$account for the initial stress at the points where the direction of the shear deformation is reversed.

Using the experimental initial conditions, i.e., $\sigma=0\left(A_{+}=\right.$ 1) at $u=0$ for $d u>0$ at point $\mathrm{A}$, the interpolation of the experimental data from A to B to Eq. (1) leads to $\sigma_{\infty}=(37 \pm 2) \mathrm{Pa}$ and a first estimate of the shear modulus, $G=(310 \pm 30) \mathrm{Pa}$. Notice that this value is consistent with the previous value of the storage shear modulus measured by shearing periodically the same material at small amplitude and frequency [19].

We can further characterize the strain-stress cycles by investigating the evolution of stress $\sigma_{\mathrm{C}}$ at point $\mathrm{C}$ [see Fig. 1(b)] as a function of the maximum imposed deformation $u_{m}$, or of the number of cycles $n$. To do so, the evolution of the constants $A_{ \pm}$can be determined throughout the cycles with the initial condition that $A_{+}^{0}=1$ in A. Simple algebra leads to, in $\mathrm{C}$ for the end of the $n$th cycle,

$$
\sigma_{\mathrm{C}}^{n}=\sigma_{\mathrm{C}}^{\infty}\left[1-\exp \left(-2 \frac{G u_{m}}{\sigma_{\infty}} n\right)\right],
$$

which tends to the asymptotic value

$$
\sigma_{\mathrm{C}}^{\infty}=-\sigma_{\infty} \tanh \left(\frac{G u_{m}}{2 \sigma_{\infty}}\right)
$$

for the limit cycle. In Fig. 2(b), the experimental values of $\sigma_{\mathrm{C}}^{\infty}$ are reported as a function of the maximum shear $u_{m}$ and fitted to Eq. (3), using $G$ and $\sigma_{\infty}$ as adjustable parameters. A good agreement is obtained, which provides second estimates of $\sigma_{\infty}=(39 \pm 3) \mathrm{Pa}$ and $G=(225 \pm 50) \mathrm{Pa}$.

As a final test of consistency, we follow the convergence of the minimum stress $\sigma_{\mathrm{C}}$ toward $\sigma_{\mathrm{C}}^{\infty}$ through the cycles. For a given amplitude $u_{m}$, Eq. (2) predicts an exponential evolution of $\sigma_{\mathrm{C}}$ with the number of cycles, $n$. We report in Fig. 2(c) the experimental and theoretical [Eq. (2)] values of $\sigma_{\mathrm{C}}^{n}$ as function of $n$ using the values of $\sigma_{\infty}$ and $G$ obtained above. In spite of large experimental errors, the observed agreement, which is fairly good agreement without further adjustment, corroborates the proposed rheological rule.

\section{MODELING AT THE MICROSCALE}

In the following we attempt to explain the rheological behavior of the material at the microscopic level. The microrheology of dry soap foams subjected to quasistatic simple shearing has been analyzed numerically by Reinelt and Kraynik [25]. Due to the relatively reduced system size, discontinuities are observed in the stress strain response, which corresponds to large changes in foam geometry and topology both resulting from unstable configurations that violate Plateau's laws. As suggested by these authors, an individual stress relaxation process would be a $T 1$ cascade, which produces different cell neighbors, reduces the surface energy of the foam, and provides mechanism for its plastic yield behavior [25]. More recently, Evans and co-workers [26] corroborated numerically that, in a $3 d$ foam, the first $T 1$ event is responsible for the elastic limit whereas "frequent $T 1$ avalanches sustain the yield-stress plateau at large scale."

Adopting a probabilistic point of view, we assume that the susceptibility of the material to the plastic deformation can be accounted for by the probability density $Q(\sigma)$ such that $Q(\sigma) d \sigma$ is the probability for any configuration of bubbles in the bulk of the material to be subjected to an irreversible change in response to an increment $d \sigma$ of the imposed shear stress. Although $Q(\sigma)$ can be any function of the stress $\sigma$, $Q(\sigma)$ is more likely to be widely distributed in the range $\sigma$ to $\sigma_{\infty}$ where, we remind, $\sigma$ is the imposed shear stress.

From now on, we assume that any rearrangement of a local configuration of bubbles leads to a local displacement $\alpha \frac{\sigma}{G} d$, proportional to the applied stress $\sigma$ and to the grain diameter $d$, which is associated with a release of a fraction $\alpha$ of the local elastic stress. Once regarded in comparison with the thickness $h$ of the sample, this displacement corresponds to a plastic deformation $d v=\alpha \frac{\sigma}{G} \frac{d}{h}$. We thus get, taking into account the number of bubbles $h / d$ in the thickness $h$ of the sample, that an increment $d \sigma$ of the shear stress leads to an increment $d v=$ $\alpha \frac{\sigma}{G} Q(\sigma) d \sigma$ in the plastic strain due to the effects of all the 
rearrangements that occur in the thickness. In consequence, the associated increment in the total shear strain, $d u \equiv d \epsilon+d v$, satisfies

$$
d u=\frac{1}{G}[1+\alpha \sigma Q(\sigma)] d \sigma .
$$

In order to obtain an expression of $Q(\sigma)$ compatible with the experimental results, let us consider Eq. (1) and note that, by differentiation, $d \sigma=G \frac{\sigma_{\infty}-\sigma}{\sigma_{\infty}} d u$. Using Eq. (4), we get

$$
Q(\sigma)=\frac{1}{\alpha} \frac{1}{\sigma_{\infty}-\sigma} .
$$

We thus get that $Q(\sigma)$ is indeed broad and that the probability of the rearrangements only depends on the distance to the yield.

The function $Q(\sigma)$ is intimately related to the stability of the local configurations of the bubbles in the bulk of the material. Let us denote $P_{\sigma}\left(\sigma^{\prime}\right)\left(\sigma^{\prime} \in\left[\sigma, \sigma_{\infty}[)\right.\right.$, the probability distribution of the stress $\sigma^{\prime}$ that the local configurations can sustain in the bulk of the sample subjected to the external stress $\sigma$. With this definition, we have $Q(\sigma)=P_{\sigma}(\sigma)$. Considering the evolution of $P_{\sigma}\left(\sigma^{\prime}\right)$ upon increase of the applied stress $\sigma$, we can write $\frac{\partial}{\partial \sigma} P_{\sigma}\left(\sigma^{\prime}\right)=p\left(\sigma \rightarrow \sigma^{\prime}\right) Q(\sigma)$ where $p\left(\sigma \rightarrow \sigma^{\prime}\right)$ stands for the probability for a local configuration that loses stability at $\sigma$ stabilizes in a new configuration that can sustain $\sigma^{\prime}$. By integration from 0 to $\sigma$, we get the condition that $Q\left(\sigma^{\prime}\right)=$ $P_{\sigma^{\prime}}\left(\sigma^{\prime}\right)=P_{0}\left(\sigma^{\prime}\right)+\int_{0}^{\sigma^{\prime}} p\left(\sigma \rightarrow \sigma^{\prime}\right) Q(\sigma) d \sigma$. The simple solution for $p\left(\sigma \rightarrow \sigma^{\prime}\right)$ is

$$
p\left(\sigma \rightarrow \sigma^{\prime}\right)=\frac{1}{\left(\sigma_{\infty}-\sigma\right)},
$$

which, interestingly, does not depend on the final state $\sigma^{\prime}$, but only on the external applied stress $\sigma$. In this picture, when a local rearrangement occurs, the bubbles locally find a stable state with equal probability in the whole accessible range $] \sigma, \sigma_{\infty}[$. Thus, as expected, the distribution $P_{\sigma}\left(\sigma^{\prime}\right)$, initially broad in a disordered system, remains as broad as possible, but narrows as deformation progresses [27].

\section{DISCUSSION AND CONCLUSION}

It is first particularly interesting to comment on the critical strain $u_{c}$, or equivalently critical stress $\sigma_{c} \equiv G u_{c}$, that leads to the first irreversible rearrangement in the thickness of the sample. Considering the probability for one rearrangement to occur in the whole thickness of the sample when the external stress is increased from 0 to $\sigma$, we have $\int_{0}^{\sigma_{c}} \frac{h}{d} Q\left(\sigma^{\prime}\right) d \sigma^{\prime}=1$. From Eq. (5), we have $\sigma_{c} \sim \alpha \frac{d}{h} \sigma_{\infty}$ or, equivalently $u_{c}=\frac{d}{h} \frac{\sigma_{\infty}}{G}$. Thus, this plasticity threshold depends on the size of the system but $u_{c}$ remains small for large systems $\left(\frac{d}{h} \ll 1\right)$. Typically, for a sample $1 \mathrm{~mm}$ thick and bubbles typically $50 \mu \mathrm{m}$ in diameter, the critical strain is of the order $u_{c} \sim 5 \times 10^{-4}$. This value of the shear strain is very small, which indicates that the system is never in the pure elastic regime and that plasticity is always at play in practice. We notice that the critical strain is experimentally unrealizable, given the reduced size of the system required, however, it seems totally accessible in numerical simulation. Indeed, $3 d$ numerical simulations in foams up to $8^{3}$ cells indicate that relative fluctuations in the shear stress during deformation scale as $d / h$ in agreement with our estimate [see Fig. 3(a) in Ref. [26]].

In conclusion, we have tested the response of foam to large amplitude shear deformations in the quasistatic regime. A simple elastoplastic model suggests that, in such disordered systems, plasticity is always at play and that the progressive hardening upon periodic shear is compatible with a very broad distribution of the stress that the internal configurations of the bubbles can sustain.

\section{ACKNOWLEDGMENTS}

The authors acknowledge the support from LIA-MSD, CNRS-France, and Conicyt-Chile through the program Fondecyt Anillo ACT-1410. F.M. acknowledges support from "Proyecto Fortalecimiento Usach USA1799-MF072215" and thanks the Ecole Normale Supérieure de Lyon for the part of the present research he conducted in Lyon as part of the guest professors program in 2018.
[1] A. S. Argon, Acta Metall. 27, 47 (1979).

[2] D. Espíndola, B. Galaz, and F. Melo, Phys. Rev. Lett 109, 158301 (2012).

[3] K. M. Schmoller, P. Fernandez, R. C. Arevalo, D. L. Blair, and A. R. Bausch, Nat. Commun. 1, 134 (2010).

[4] K. M. Schmoller and A. R. Bausch, Nat. Mater. 12, 278 (2013).

[5] L. Mullins, Rubber Chem. Technol. 21, 281 (1948).

[6] L. Mullins, Rubber Chem. Technol. 42, 339 (1969).

[7] M. Gralka and K. Kroy, Biochim. Biophys. Acta, Mol. Cell Res. 1853, 3025 (2015).

[8] G. Liu, S. Cheng, H. Lee, H. Ma, H. Xu, T. Chang, R. P Quirk, and S.-Q. Wang, Phys. Rev. Lett. 111, 068302 (2013)

[9] V. B. Nguyen, T. Darnige, A. Bruand, and E. Clément, Phys. Rev. Lett. 107, 138303 (2011).

[10] F. Rouyer, S. Cohen-Addad, R. Hohler, P. Sollich, and S. M. Fielding, Eur. Phys. J. E 27, 309 (2008).

[11] G. Marckmann et al., J. Mech. Phys. Solids. 50, 2011 (2002).
[12] J. Diani, B. Fayolle, and P. Gilormini, Eur. Polym. J. 45, 601 (2009).

[13] L. Bocquet and A. Colin, and A. Ajdari, Phys. Rev. Lett. 103, 036001 (2009).

[14] J. Lauridsen and M. Twardos, and M. Dennin, Phys. Rev. Lett. 89, 098303 (2002).

[15] G. Debrégeas, H. Tabuteau, and J.-M. di Meglio, Phys. Rev. Lett. 87, 178305 (2001).

[16] G. Katgert, M. E. Mobius, and M. van Hecke, Phys. Rev. Lett. 101, 058301 (2008).

[17] E. Janiaud, D. Weaire, and S. Hutzler, Phys. Rev. Lett. 97, 038302 (2006).

[18] N. D. Denkov, S. Tcholakova, K. Golemanov, and A. Lips, Phys. Rev. Lett. 103, 118302 (2009).

[19] S. Cohen-Addad, H. Hoballah, and R. Hohler, Phys. Rev. E 57, 6897 (1998).

[20] J. C. Germain and J. M. Aguilera, Food Struct. 1, 55 (2014). 
[21] I. Cantat, S. Cohen-Addad, F. Elias, F. Graner, R. Höhler, O. Pitois, F. Rouyer, and A. Saint-Jalmes, Foams: Structure and Dynamics (Oxford University Press, Oxford, 2013).

[22] D. Weaire and M. A. Fortes, Adv. Phys. 43, 685 (1994).

[23] D. Weaire and J. P. Kermode, Philos. Mag. B 48, 245 (1983).
[24] D. Weaire and J. P. Kermode, Philos. Mag. B 50, 379 (1984).

[25] D. A. Reinelt and A. M. Kraynik, J. Rheol. 44, 453 (2000).

[26] M. E. Evans, A. M. Kraynik, D. A. Reinelt, K. Mecke, and G. E. Schröder-Turk, Phys. Rev. Lett. 111, 138301 (2013).

[27] P. Marmottant and F. Graner, Eur. Phys. J. E 23, 337 (2007). 